\title{
Nonlinear viscosity and velocity distribution function in a simple longitudinal flow
}

\author{
Andrés Santos* \\ Departamento de Física, Universidad de Extremadura, \\ E-06071 Badajoz, Spain
}

(October 27, 2018)

\begin{abstract}
A compressible flow characterized by a velocity field $u_{x}(x, t)=a x /(1+a t)$ is analyzed by means of the Boltzmann equation and the Bhatnagar-Gross-Krook kinetic model. The sign of the control parameter (the longitudinal deformation rate $a$ ) distinguishes between an expansion $(a>0)$ and a condensation $(a<0)$ phenomenon. The temperature is a decreasing function of time in the former case, while it is an increasing function in the latter. The non-Newtonian behavior of the gas is described by a dimensionless nonlinear viscosity $\eta^{*}\left(a^{*}\right)$, that depends on the dimensionless longitudinal rate $a^{*}$. The Chapman-Enskog expansion of $\eta^{*}$ in powers of $a^{*}$ is seen to be only asymptotic (except in the case of Maxwell molecules). The velocity distribution function is also studied. At any value of $a^{*}$, it exhibits an algebraic high-velocity tail that is responsible for the divergence of velocity moments. For sufficiently negative $a^{*}$, moments of degree four and higher may diverge, while for positive $a^{*}$ the divergence occurs in moments of degree equal to or larger than eight.
\end{abstract}

PACS numbers: 05.20.Dd, 47.50.+d, 05.60.-k, 51.10.+y

\section{INTRODUCTION}

One of the most challenging problems in nonequilibrium statistical mechanics is the understanding of transport properties in fluids beyond the scope of the Navier-Stokes (NS) constitutive equations. As part of the NS constitutive equations, Newton's law establishes a linear relationship between the irreversible momentum flux and the velocity gradients, namely

$$
P_{i j}=p \delta_{i j}-\eta_{\mathrm{NS}}\left(\frac{\partial u_{i}}{\partial x_{j}}+\frac{\partial u_{j}}{\partial x_{i}}-\frac{2}{d} \nabla \cdot \mathbf{u} \delta_{i j}\right)-\zeta_{\mathrm{NS}} \nabla \cdot \mathbf{u} \delta_{i j},
$$

where $P_{i j}$ is the pressure tensor, $p=(1 / d) \operatorname{Tr} \mathrm{P}$ is the hydrostatic pressure, $d \geq 2$ being the dimensionality of the system, $\mathbf{u}$ is the flow velocity, $\eta_{\mathrm{NS}}$ is the shear viscosity, and $\zeta_{\mathrm{NS}}$ is the bulk viscosity [1]. The linear law (1) only holds for small hydrodynamic gradients, i.e., when the typical distances over which the hydrodynamic quantities change are much larger than a characteristic microscopic length (such as the mean free path in the case of gases). Otherwise, Eq. (1) no longer holds, a situation usually characterized by the introduction of a generalized or nonlinear viscosity that depends on the hydrodynamic gradients [2].

The nonlinear viscosity has been extensively studied in the so-called uniform shear flow, which is characterized by a linear velocity field $u_{x}=a y$ and uniform density and temperature [3-7]. This is an example of an incompressible flow [1], since $\nabla \cdot \mathbf{u}=0$. Recently, some attention has been devoted to viscous longitudinal flows of the form $\mathbf{u}(\mathbf{r}, t)=u_{x}(x, t) \widehat{\mathbf{x}}[12]$. The simplest example of such compressible flows is characterized by a linear velocity profile, i.e., $u_{x}(x, t)=A(t) x$, and uniform density $n$ and pressure tensor $\mathrm{P}$ 13.14. In that case, the balance equations for mass and momentum read

$$
\frac{\partial n}{\partial t}=-n A, \quad \frac{\partial(n A)}{\partial t}=-2 n A^{2},
$$

whose solution is

$$
A(t)=\frac{a}{1+a t}, \quad n(t)=\frac{n_{0}}{1+a t},
$$

where $a$ is an arbitrary constant that represents the (initial) longitudinal deformation rate and $n_{0}>0$ is the initial density. In this case, Newton's law (1i) becomes

$$
P_{x x}=p-\left(2 \frac{d-1}{d} \eta_{\mathrm{NS}}+\zeta_{\mathrm{NS}}\right) A .
$$


This simple flow is known as homo-energetic extension and, along with the uniform shear flow, is a particular case of a more general class of homo-energetic affine flows characterized by $\partial^{2} u_{i} / \partial x_{j} \partial x_{k}=0$ [13]. In the flow defined by Eqs. (3) the longitudinal deformation rate $a$ is the only control parameter determining the departure of the fluid from its equilibrium state, thus playing a role similar to that of the shear rate in the uniform shear flow state. On the other hand, in contrast to the uniform shear flow, the sign of $a$ plays a relevant role and defines two distinct situations. The case $a>0$ corresponds to a progressively more slowly expansion of the gas from the plane $x=0$ into all of space. Given a layer of width $\delta$, the flux of particles leaving the layer exceeds the flux of incoming particles by $n a \delta /(1+a t)$ and, as a consequence, the number of particles inside the layer decreases monotonically with time. As time progresses, the system becomes more and more rarefied until no particles are left in the long-time limit, i.e., $\lim _{t \rightarrow \infty} n(t)=0$. On the other hand, the case $a<0$ corresponds to a progressively more rapidly condensation of the gas towards the plane $x=0$. The latter takes place over a finite time period $t=|a|^{-1}$. However, since the collision frequency rapidly increases with time, the finite period $t=|a|^{-1}$ comprises an infinite number of collisions per particle (see below).

Equations (3) apply regardless of the initial density of the fluid. On the other hand, a kinetic description based on the Boltzmann equation is valid only for densities such that $n \sigma^{d}$ is much smaller than 1 , where $\sigma$ is a characteristic distance measuring the effective size of the molecules. Let us call $n_{\sigma} \sim \sigma^{-d}$ a characteristic density beyond which noticeable deviations from the Boltzmann equation can be expected. Thus, even if $n_{0} \ll n_{\sigma}$, there exists a finite time $t_{\sigma}=|a|^{-1}\left(1-n_{0} / n_{\sigma}\right)$ beyond which the Boltzmann description ceases to be applicable in the case $a<0$. This time $t_{\sigma}$ can be made arbitrarily close to the maximum time $|a|^{-1}$ by formally taking the limit $n_{0} / n_{\sigma} \rightarrow 0$.

The aim of this paper is to carry out a detailed and self-contained analysis of the nonequilibrium behavior of a dilute gas under the longitudinal flow characterized by Eqs. (3), for arbitrary sign and magnitude of the control parameter $a$. The study is performed by using the tools of kinetic theory, namely the Boltzmann equation and the Bhatnagar-Gross-Krook (BGK) kinetic model, and deals with the nonlinear viscosity, as well as with more general velocity moments and the velocity distribution function. Most of the results are derived for arbitrary dimensionality and for the general class of repulsive potentials of the form $r^{-\mu}$ with $\mu \geq 2(d-1)$. The Boltzmann equation for the problem is considered in Sec. [1]. Since the density is known, cf. Eqs. (3), one can focus on the probability distribution of velocities. In addition, the distribution becomes uniform when the velocities are referred to the local Lagrangian frame moving with the flow velocity $u_{x}(x, t)$. As a consequence, the original problem can be mapped onto that of a uniform system with a stationary density and subject to the action of a non-conservative driving force; also, there is a nonlinear relationship between the time variables in the original and the equivalent systems. To proceed further, the Maxwell interaction, $\mu=2(d-1)$, is assumed and the time evolution of the pressure tensor is exactly obtained. The long-time behavior allows one to identify the nonlinear viscosity as a function of the longitudinal deformation rate. When the velocities are scaled with the (time-dependent) thermal velocity, the distribution obeys in the long-time limit a steady-state Boltzmann equation with the addition of a second non-conservative force playing the role of a thermostat. The exact fourth-degree velocity moments are then derived as functions of the longitudinal rate and are seen to diverge in the case of condensation for states sufficiently far from equilibrium. The picture is complemented in Sec. III by the solution of the BGK kinetic model. In the case of Maxwell molecules, the distribution function exhibits an algebraic high-velocity tail that is responsible for the divergence of the moments. The solution predicts that moments of sufficiently high degree can also diverge in the case of expansion. In addition, the distribution function becomes infinite in the limit of zero velocity in far from equilibrium states. Section III also analyzes the nonlinear viscosity for more general repulsive potentials, including hard spheres. Evidence is given about the non-convergent (but asymptotic) character of the Chapman-Enskog expansion, Maxwell molecules being an exception. On the other hand, the nonlinear dependence of the generalized viscosity is practically insensitive to the interaction potential in the case of positive deformation rates. For negative rates, however, the influence of the potential is not small, especially near the maximum value of the viscosity. The paper ends with a summary of the main conclusions in Sec. IV.

\section{BOLTZMANN DESCRIPTION FOR MAXWELL MOLECULES}

Let us consider a dilute gas subject to the homo-energetic extension flow described in the previous Section. All the relevant information is contained in the one-particle velocity distribution function $f(x, \mathbf{v}, t)$. In particular, the number density $n$, the flow velocity $\mathbf{u}$, and the pressure tensor $\mathrm{P}$ are obtained in terms of velocity moments of $f$,

$$
n=\int \mathrm{d} \mathbf{v} f, \quad n \mathbf{u}=\int \mathrm{d} \mathbf{v} \mathbf{v} f, \quad \mathrm{P}=m \int \mathrm{d} \mathbf{v} \mathbf{V} \mathbf{V} f .
$$

Here $m$ is the mass of a particle and $\mathbf{V} \equiv \mathbf{v}-\mathbf{u}$ is the peculiar velocity. The trace of $\mathbf{P}$ gives the hydrostatic pressure $p=(1 / d) \operatorname{Tr} \mathrm{P}$, which is related to the temperature $T$ through the equation of state $p=n k_{B} T, k_{B}$ being the Boltzmann constant. The time evolution of $f$ is governed by the Boltzmann equation 15 


$$
\left(\frac{\partial}{\partial t}+v_{x} \frac{\partial}{\partial x}\right) f=J[f, f]
$$

where $J[f, f]$ is the nonlinear Boltzmann collision operator, whose explicit expression will be omitted here.

As happens in the uniform shear flow [3. 16], the velocity distribution function $f(x, \mathbf{v}, t)$ becomes spatially uniform when the velocities are referred to a Lagrangian frame moving with the flow, i.e., $f(x, \mathbf{v}, t)=f(\mathbf{V}, t)$, where $\mathbf{V} \equiv$ $\mathbf{v}-\mathbf{u}(x, t)$ is the peculiar velocity. The Boltzmann equation (6) for this flow can then be written as

$$
\left(\frac{\partial}{\partial \tau}-a \frac{\partial}{\partial V_{x}} V_{x}\right) \tilde{f}=J[\tilde{f}, \tilde{f}]
$$

where

$$
\widetilde{f}(\mathbf{V}, \tau)=\frac{n_{0}}{n(t)} f(x, \mathbf{v}, t), \quad \tau=a^{-1} \ln (1+a t) .
$$

Equation (7) can be interpreted as corresponding to a homogeneous gas with a velocity distribution $\tilde{f}$ and subject to the action of a non-conservative force $-m a V_{x} \widehat{\mathbf{x}}$. Note that the density and pressure tensor associated with $\tilde{f}$ are $\widetilde{n}=n_{0}$ and $\widetilde{P}_{i j}=\left(n_{0} / n\right) P_{i j}$, respectively. In fact, $\widetilde{f}$ is proportional to the probability distribution of velocities. The time variable $\tau=\int_{0}^{t} \mathrm{~d} t^{\prime} n\left(t^{\prime}\right) / n_{0}$ is a nonlinear measure of time scaled with the number density; this variable is unbounded even if $a<0$, since in that case $\tau \rightarrow \infty$ when $t \rightarrow|a|^{-1}$. It must be emphasized that Eqs. (6) and (7) are fully equivalent in the present problem. On the other hand, Eq. (7) has the advantage of describing a uniform system with a constant density. The prize to be paid is the introduction of a driving force that acts as a longitudinal "drag" force in the case of expansion $(a>0)$ and as a "pushing" force in the case of condensation $(a<0)$. Of course, every solution to Eq. (7) can be mapped onto a corresponding solution to Eq. (6).

The Boltzmann equation (f) cannot be solved by analytical tools in general. On the other hand, its associated hierarchy of moment equations can be recursively solved in the special case of Maxwell molecules, i.e., particles interacting via a repulsive potential of the form $\phi(r) \propto r^{-2(d-1)}$, in which case the collision rate is independent of the relative velocity of the colliding particles [17]. In particular, Eq. (7) yields a closed set of equations for the elements of the pressure tensor in the case of Maxwell molecules, namely

$$
\begin{gathered}
\frac{\partial}{\partial \tau} \widetilde{p}+\frac{2 a}{d} \widetilde{P}_{x x}=0 \\
\frac{\partial}{\partial \tau} \widetilde{P}_{x x}+2 a \widetilde{P}_{x x}=-\nu_{0}\left(\widetilde{P}_{x x}-\widetilde{p}\right),
\end{gathered}
$$

where $\widetilde{p}=n_{0} k_{B} T=(1 / d) \operatorname{Tr} \widetilde{\mathrm{P}}$ and $\nu_{0} \propto n_{0}$ is a constant that plays the role of an effective (initial) collision frequency. More explicitly, $\nu_{0}=\widetilde{p} / \eta_{\mathrm{NS}}$. Equation (9) is not but a condition expressing the conservation of energy. As for Eq. (10), it must be emphasized that it is exact for Maxwell molecules in our problem, i.e., no approximate truncation scheme (such as Grad's moment method [12]) has been applied. This is a consequence of the fact that the collisional velocity moments of a certain degree do not involve moments of a higher degree in the case of the Maxwell interaction [13]. The right-hand-side of Eq. (10) represents the (bilinear) collisional moment of $V_{x}^{2}$.

In this case of Maxwell molecules the time variable $\tau$ is just proportional to the average number of collisions per particle between 0 and $t$, namely $\tau=\nu_{0}^{-1} \int_{0}^{t} \mathrm{~d} t^{\prime} \nu\left(t^{\prime}\right)$, where $\nu=p / \eta_{\mathrm{NS}}=\left(n / n_{0}\right) \nu_{0}$ is the time-dependent collision frequency. Thus, as said in Sec. I, every particle experiences an infinite total number of collisions between the initial time and the finite interval $t=|a|^{-1}$ when $a<0$. After many collision times $\left(\tau \gg \nu_{0}^{-1}\right)$ both $\widetilde{p}$ and $\widetilde{P}_{x x}$ behave as $\widetilde{p}, \widetilde{P}_{x x} \sim \exp \left[-\lambda\left(a^{*}\right) \nu_{0} \tau\right]$, where $a^{*} \equiv a / \nu_{0}=A(t) / \nu(t)$ is the reduced longitudinal rate and $\lambda\left(a^{*}\right)$ is the smallest root of the quadratic equation

$$
\lambda^{2}-\left(2 a^{*}+1\right) \lambda+\frac{2}{d} a^{*}=0
$$

i.e.,

$$
\lambda\left(a^{*}\right)=a^{*}+\frac{1}{2}-\sqrt{\left(a^{*}+\frac{1}{2}\right)^{2}-\frac{2}{d} a^{*}}
$$


The second root is obtained from (12) by changing the sign of the radical and is only relevant in the transient stage. Consequently,

$$
\lim _{\tau \rightarrow \infty} \frac{\widetilde{P}_{x x}}{\widetilde{p}}=\frac{d}{2} \frac{\lambda\left(a^{*}\right)}{a^{*}}
$$

In terms of the real time, one has an algebraic behavior for the temperature, $T(t) \sim(1+a t)^{-\lambda\left(a^{*}\right) / a^{*}}$. It is important to note that the sign of $\lambda$ is the same as that of $a^{*}$. This means that the temperature monotonically decreases in time if $a^{*}>0$ (expansion, drag force), while it increases if $a^{*}<0$ (condensation, pushing force). In addition, $\lambda$ is a monotonically increasing function of $a^{*}$ that behaves as $\lambda \approx 2 a^{*}+(d-1) / d$ in the limit $a^{*} \rightarrow-\infty$ and as $\lambda \approx\left[1-(d-1) / 2 d a^{*}\right] / d$ in the limit $a^{*} \rightarrow \infty$.

Based upon Eq. (4), we define in the long-time limit a (dimensionless) nonlinear viscosity as [12]

$$
\begin{aligned}
\eta^{*} & =\frac{d}{2(d-1)} \frac{p-P_{x x}}{A \eta_{\mathrm{NS}}} \\
& =\frac{d}{2(d-1) a^{*}}\left(1-\frac{P_{x x}}{p}\right),
\end{aligned}
$$

where we have taken into account that the bulk viscosity $\zeta_{\mathrm{NS}}$ vanishes in a low-density gas 115 . Using Eq. (13), we simply get

$$
\eta^{*}\left(a^{*}\right)=\frac{d}{d-1} \frac{2 a^{*}-d \lambda\left(a^{*}\right)}{4 a^{* 2}} .
$$

In the three-dimensional case $(d=3)$, this result coincides with the one derived by Karlin et al. [9] for Maxwell molecules by applying the invariance principle under the microscopic and macroscopic dynamics in the context of Grad's method. It should also be noted that, although by a somewhat different route, most of the above results (for $d=3$ ) were first derived by Galkin more than thirty years ago 13,14$]$.

The behavior of the nonlinear viscosity (15) for small longitudinal rates is $\eta^{*} \approx 1-[2(d-2) / d] a^{*}$. More generally, $\eta^{*}$ can be expressed as a series expansion in powers of $a^{*}$ :

$$
\eta^{*}\left(a^{*}\right)=1+\sum_{n=1}^{\infty} c_{n} a^{* n} .
$$

This is just the specialization of the Chapman-Enskog expansion [15, 18] to the simple viscous longitudinal flow. In the case of Maxwell molecules, $a^{*}=0$ is a regular point of $\eta^{*}$, so the expansion (16) is convergent, although with a finite radius of convergence $\left(\left|a^{*}\right|<\frac{1}{2}\right)$ due to a branch point at $a^{*}=-(d-2) / 2 d \pm \imath \sqrt{d-1} / d$. The knowledge of the explicit expression of $\eta^{*}$, Eq. (15), allows one to get also its asymptotic behaviors for large $\left|a^{*}\right|$; they are $\eta^{*} \approx[d / 2(d-1)] a^{*-1}$ for $a^{*}>0$ and $\eta^{*} \approx(d / 2)\left|a^{*}\right|^{-1}$ for $a^{*}<0$. This implies that $P_{x x} / p \rightarrow 0$ when $a^{*} \rightarrow+\infty$, while $P_{x x} / p \rightarrow d$ when $a^{*} \rightarrow-\infty$. In the former limit all the particles tend to move along the transverse directions, while in the latter they tend to move along the longitudinal direction. The shape of $\eta^{*}\left(a^{*}\right)$ for $d=3$ in the range $-2 \leq a^{*} \leq 2$ is shown in the next Section (cf. Fig. 司).

Because of the symmetry of the problem, one expects that the heat flux is an irrelevant quantity [12] that, even if it is initially different from zero, asymptotically decays in the long-time limit. Let us analyze this point more carefully. Taking third-degree moments in Eq. (7), we get

$$
\begin{gathered}
\frac{\partial}{\partial \tau} \widetilde{q}_{x}+a\left(\widetilde{q}_{x}+2 \widetilde{M}_{x x x}\right)=-\frac{2}{3} \nu_{0} \widetilde{q}_{x}, \\
\frac{\partial}{\partial \tau} \widetilde{M}_{x x x}+3 a \widetilde{M}_{x x x}=-\nu_{0}\left(\frac{3}{2} \widetilde{M}_{x x x}-\frac{1}{2} \widetilde{q}_{x}\right),
\end{gathered}
$$

where

$$
\widetilde{q}_{x}=\frac{m}{2} \int \mathrm{d} \mathbf{V} V^{2} V_{x} \widetilde{f}, \quad \widetilde{M}_{x x x}=\frac{m}{2} \int \mathrm{d} \mathbf{V} V_{x}^{3} \widetilde{f} .
$$

In Eqs. (17) and (18) use has been made of the third-degree collisional moments for the three-dimensional Maxwell interaction [13]. For long collision times $\left(\tau \gg \nu_{0}^{-1}\right)$, the third-degree moments behave as $\widetilde{q}_{x}, \widetilde{M}_{x x x} \sim \exp \left[-\omega\left(a^{*}\right) \nu_{0} \tau\right]$, 
where $\omega\left(a^{*}\right) \equiv 2 a^{*}+\frac{13}{12}-\sqrt{\left(a^{*}+\frac{5}{12}\right)^{2}-a^{*}}$. If $a^{*}<-\frac{3}{4}+\frac{\sqrt{33}}{12} \simeq-0.271, \omega$ is negative and then the heat flux grows in time. Apparently, this seems to contradict our expectation about the irrelevance of the heat flux in our problem. The solution to this paradox lies in the fact that also the temperature grows if $a^{*}<0$; indeed, what is relevant is not the absolute value of the heat flux but its value relative to the third power of the thermal velocity, namely $\widetilde{q}_{x} / m n_{0}\left(2 k_{B} T / m\right)^{3 / 2} \sim \exp \left\{-\left[\omega\left(a^{*}\right)-\frac{3}{2} \lambda\left(a^{*}\right)\right] \nu_{0} \tau\right\}$. The difference $\omega\left(a^{*}\right)-\frac{3}{2} \lambda\left(a^{*}\right)$ is always positive, but goes to zero as $\frac{1}{12}\left|a^{*}\right|^{-1}$ in the limit $a^{*} \rightarrow-\infty$. In general, the smaller the value of $a^{*}$ the longer the transient period before the heat flux, conveniently scaled with the thermal velocity, has decayed to zero.

In the long-time limit, not only the reduced elements of the pressure tensor $P_{i j} / p$ reach well-defined stationary values that depend on the reduced longitudinal rate $a^{*}$, but the same happens with the distribution function when properly nondimensionalized with the temperature. This is just a statement on the validity of the "normal" or hydrodynamic regime, that applies for sufficiently long times [15,18. To be more precise, let us introduce a reduced velocity $\boldsymbol{\xi}$ and a reduced distribution function $\Phi$ as

$$
\boldsymbol{\xi}=\left[\frac{m}{2 k_{B} T(\tau)}\right]^{1 / 2} \mathbf{V}, \quad \Phi\left(\boldsymbol{\xi} ; a^{*}\right)=n_{0}^{-1} \lim _{\tau \rightarrow \infty}\left[\frac{2 k_{B} T(\tau)}{m}\right]^{d / 2} \tilde{f}(\mathbf{V}, \tau)
$$

In this hydrodynamic regime, the Boltzmann equation (7) becomes (for Maxwell molecules)

$$
\left(-a^{*} \frac{\partial}{\partial \xi_{x}} \xi_{x}+\frac{\lambda\left(a^{*}\right)}{2} \frac{\partial}{\partial \boldsymbol{\xi}} \cdot \boldsymbol{\xi}\right) \Phi=\frac{1}{\nu_{0}} J[\Phi, \Phi] .
$$

As before, the first term on the left side represents a driving force. The second term can be interpreted as a thermostat force [3, that compensates for the heating $\left(a^{*}<0\right)$ or cooling $\left(a^{*}>0\right)$ effect produced by the former. Equation (21) yields directly the "stationary" values of the (reduced) second-degree moments, namely

$$
\left\langle\xi_{x}^{2}\right\rangle=\frac{1}{2} \frac{1}{1+2 a^{*}-\lambda}, \quad\left\langle\xi_{\perp}^{2}\right\rangle=\frac{d-1}{2} \frac{1}{1-\lambda},
$$

where $\xi_{\perp}^{2}=\xi^{2}-\xi_{x}^{2}$. The consistency condition $\left\langle\xi^{2}\right\rangle=d / 2$ leads again to Eq. (11). As noted before, $\left\langle\xi_{x}^{2}\right\rangle \rightarrow 0$ in the limit $a^{*} \rightarrow+\infty$ and $\left\langle\xi_{\perp}^{2}\right\rangle \rightarrow 0$ in the opposite limit $a^{*} \rightarrow-\infty$. This means that all the particles move (in the Lagrangian frame) along directions perpendicular to the flow when $a^{*} \rightarrow+\infty$, while they move parallel to the flow direction when $a^{*} \rightarrow-\infty$.

Now we can go further and consider the fourth-degree moments $\left\langle\xi_{x}^{4}\right\rangle,\left\langle\xi_{x}^{2} \xi_{\perp}^{2}\right\rangle$, and $\left\langle\xi_{\perp}^{4}\right\rangle$. Making use of the fourthdegree collisional moments for three-dimensional Maxwell molecules [13], Eq. (21) gives rise to

$$
\begin{aligned}
2\left(2 a^{*}-\lambda\right)\left\langle\xi_{x}^{4}\right\rangle= & -\frac{4(2 w+7)}{35}\left\langle\xi_{x}^{4}\right\rangle+\frac{3(8 w-7)}{35}\left\langle\xi_{x}^{2} \xi_{\perp}^{2}\right\rangle+\frac{7-3 w}{35}\left\langle\xi_{\perp}^{4}\right\rangle \\
& -\frac{54 w-91}{35}\left\langle\xi_{x}^{2}\right\rangle^{2}+\frac{3(36 w-49)}{70}\left\langle\xi_{x}^{2}\right\rangle+\frac{9(7-3 w)}{70}, \\
2\left(a^{*}-\lambda\right)\left\langle\xi_{x}^{2} \xi_{\perp}^{2}\right\rangle= & -\frac{144 w+49}{210}\left\langle\xi_{x}^{2} \xi_{\perp}^{2}\right\rangle+\frac{8 w-7}{35}\left\langle\xi_{x}^{4}\right\rangle+\frac{18 w-7}{210}\left\langle\xi_{\perp}^{4}\right\rangle \\
& -\frac{343-162 w}{105}\left\langle\xi_{x}^{2}\right\rangle^{2}+\frac{469-216 w}{140}\left\langle\xi_{x}^{2}\right\rangle+\frac{3(36 w-49)}{280}, \\
-2 \lambda\left\langle\xi_{\perp}^{4}\right\rangle= & -\frac{3 w+28}{35}\left\langle\xi_{\perp}^{4}\right\rangle+\frac{8(7-3 w)}{105}\left\langle\xi_{x}^{4}\right\rangle+\frac{4(18 w-7)}{105}\left\langle\xi_{x}^{2} \xi_{\perp}^{2}\right\rangle \\
& +\frac{2(154 w-81)}{105}\left\langle\xi_{x}^{2}\right\rangle^{2}-\frac{18(7-3 w)}{35}\left\langle\xi_{x}^{2}\right\rangle+\frac{3(56-9 w)}{70},
\end{aligned}
$$

where $w \simeq 1.8731$ is the ratio of two eigenvalues of the linearized collision operator [19]. The solution of this linear set of algebraic equations is

$$
\begin{aligned}
\left\langle\xi_{x}^{4}\right\rangle= & \frac{3(1-3 \lambda)}{4 \Delta\left(a^{*}\right)}\left[-1296(7-3 w) \lambda^{5}+54(217-48 w) \lambda^{4}-9(327 w+602) \lambda^{3}\right. \\
& \left.+33(129 w+14) \lambda^{2}-25(75 w-14) \lambda+245 w\right], \\
\left\langle\xi_{x}^{2} \xi_{\perp}^{2}\right\rangle= & \frac{1-3 \lambda}{2 \Delta\left(a^{*}\right)}\left[432(7-3 w) \lambda^{4}-54(64 w-91) \lambda^{3}\right. \\
& \left.+9(363 w-392) \lambda^{2}-10(114 w-35) \lambda+245 w\right],
\end{aligned}
$$




$$
\begin{aligned}
\left\langle\xi_{\perp}^{4}\right\rangle= & \frac{2}{\Delta\left(a^{*}\right)}\left[324(7-3 w) \lambda^{4}-90(6 w+7) \lambda^{3}\right. \\
& \left.+9(143 w+28) \lambda^{2}-10(114 w-35) \lambda+245 w\right]
\end{aligned}
$$

where

$$
\begin{aligned}
\Delta\left(a^{*}\right) \equiv & (1-\lambda)^{2}\left[-432(7-3 w) \lambda^{4}-54(8 w-7) \lambda^{3}\right. \\
& \left.+9(159 w-56) \lambda^{2}-10(114 w-35) \lambda+245 w\right] .
\end{aligned}
$$

Upon writing Eqs. (26)-(29) we have made use of (22) and have eliminated $a^{*}$ in favor of $\lambda$. In the limit $a^{*} \rightarrow+\infty$, i.e., $\lambda \rightarrow \frac{1}{3}$, we have $\left\langle\xi_{\perp}^{4}\right\rangle=9(56-9 w) / 2(14 w+9) \simeq 5.71$ and $\left\langle\xi_{x}^{4}\right\rangle=\left\langle\xi_{x}^{2} \xi_{\perp}^{2}\right\rangle=0$, in agreement with a vanishing population of particles moving along the longitudinal direction. A more interesting situation occurs in the domain of negative $a^{*}$ (condensation case). As $a^{*}$ becomes more and more negative, the three moments $\left\langle\xi_{x}^{4}\right\rangle,\left\langle\xi_{x}^{2} \xi_{\perp}^{2}\right\rangle$, and $\left\langle\xi_{\perp}^{4}\right\rangle$ grow monotonically and eventually diverge when $a^{*}$ approaches a critical value $a_{c}^{*} \simeq-1.599$ (which corresponds to the root $\lambda_{c} \simeq-2.607$ of the function $\Delta$ ). This singular behavior of the fourth-degree moments also occurs in the case of uniform shear flow 20,21] and is an indication of an algebraic high-velocity tail in the distribution function 22, 23]. We will return to this point later on. The moments $\left\langle\xi_{x}^{4}\right\rangle,\left\langle\xi_{x}^{2} \xi_{\perp}^{2}\right\rangle$, and $\left\langle\xi_{\perp}^{4}\right\rangle$ are plotted in Fig. 1. While for $a^{*} \gtrsim 0.24$ one has $\left\langle\xi_{x}^{4}\right\rangle<\left\langle\xi_{x}^{2} \xi_{\perp}^{2}\right\rangle<\left\langle\xi_{\perp}^{4}\right\rangle$, the order is reversed for $a^{*} \lesssim-0.75$.

\section{KINETIC MODEL DESCRIPTION}

The description in the previous Section is based on the Boltzmann equation. It has, however, two shortcomings. On the one hand, it is restricted to Maxwell molecules. On the other hand, even for Maxwell molecules, the explicit expression for the velocity distribution function is not known. Both limitations are overcome if one resorts to a description less detailed than that offered by the Boltzmann equation and employs a model kinetic equation based on it. The simplest and best known model kinetic equation is the one proposed by Bhatnagar, Gross, and Krook (BGK) [24]. It consists of replacing the true collision operator $J[f, f]$ by a single-time relaxation term of the form $-\nu\left(f-f_{L}\right)$, where $\nu$ is an effective collision frequency and

$$
f_{L}=n\left(\frac{m}{2 \pi k_{B} T}\right)^{d / 2} \exp \left(-\frac{m V^{2}}{2 k_{B} T}\right)
$$

is the local equilibrium distribution function. The collision frequency $\nu$ is also a functional of $f$ through its dependence on the density and the temperature. While the dependence on $n$ is always linear, its dependence on $T$ varies according to the interaction potential under consideration. For instance, in the case of repulsive potentials of the form $\phi(r) \sim r^{-\mu}$, we simply have $\nu \propto n T^{\gamma}$ with $\gamma=\frac{1}{2}-(d-1) / \mu[17$. The extreme cases correspond to Maxwell molecules $(\mu=2(d-1)$, $\gamma=0)$ and hard spheres $\left(\mu \rightarrow \infty, \gamma=\frac{1}{2}\right)$. For this class of repulsive potentials, the Boltzmann equation (7) is modeled as

$$
\left(\frac{\partial}{\partial \tau}-a \frac{\partial}{\partial V_{x}} V_{x}\right) \tilde{f}=-\nu_{0}\left(T / T_{0}\right)^{\gamma}\left(\tilde{f}-\tilde{f}_{L}\right), \quad \tilde{f}_{L}=\left(n_{0} / n\right) f_{L},
$$

where, as before, the subscript 0 denotes initial values. Since the BGK model contains a single parameter $(\nu)$, it is unable to reproduce the correct Boltzmann values of both the shear viscosity an the thermal conductivity coefficients simultaneously. In our problem, however, only the shear viscosity is relevant and thus the effective collision frequency $\nu$ can be chosen as $\nu=p / \eta_{\mathrm{NS}}$, so that the exact NS viscosity is recovered.

\section{A. Maxwell molecules}

In this subsection we specialize to Maxwell molecules $(\gamma=0)$. In that case, the evolution equations for the elements of the pressure tensor are identical to those already derived from the Boltzmann equation, Eqs. (9) and (10), provided that the BGK collision frequency $\nu_{0}$ is identified with the one arising from the Boltzmann equation. As a consequence, the nonlinear viscosity is again given by Eqs. (15) and (12). However, velocity moments of degree higher than two no longer coincide in both descriptions. The main advantage of the BGK equation is that it lends itself to an exact

solution at the level of the distribution function. This solution is expected to provide a fair description of the true distribution at least for velocities smaller than or of the order of the thermal velocity $\left(2 k_{B} T / m\right)^{1 / 2}$. 
The general solution of Eq. (31) for Maxwell molecules is

$$
\begin{aligned}
\tilde{f}(\mathbf{V}, \tau)= & e^{-\nu_{0} \tau} e^{a \tau \partial_{V_{x}} V_{x}} \tilde{f}(\mathbf{V}, 0) \\
& +\nu_{0} \int_{0}^{\tau} \mathrm{d} \tau^{\prime} e^{-\nu_{0}\left(\tau-\tau^{\prime}\right)} e^{a\left(\tau-\tau^{\prime}\right) \partial_{V_{x}} V_{x}} \widetilde{f}_{L}\left(\mathbf{V}, \tau^{\prime}\right),
\end{aligned}
$$

where the action of the operator $\exp \left(a \tau \partial_{V_{x}} V_{x}\right)$ is

$$
e^{a \tau \partial_{V_{x}} V_{x}} F\left(V_{x}\right)=e^{a \tau} F\left(e^{a \tau} V_{x}\right) .
$$

We will focus on the long-time distribution function, which becomes independent of the choice of the initial distribution $\widetilde{f}(\mathbf{V}, 0)=f(\mathbf{V}, 0)$. To that end, it is convenient to work with the reduced quantities (20). The BGK counterpart of Eq. (21) is

$$
\left(-a^{*} \frac{\partial}{\partial \xi_{x}} \xi_{x}+\frac{\lambda\left(a^{*}\right)}{2} \frac{\partial}{\partial \boldsymbol{\xi}} \cdot \boldsymbol{\xi}\right) \Phi=-\Phi+\pi^{-d / 2} e^{-\xi^{2}}
$$

whose solution is

$$
\Phi\left(\boldsymbol{\xi} ; a^{*}\right)=\pi^{-d / 2} \int_{0}^{\infty} \mathrm{d} s \exp \left[-\left(1-a^{*}+\frac{d}{2} \lambda\right) s-e^{-\lambda s}\left(e^{2 a^{*} s} \xi_{x}^{2}+\xi_{\perp}^{2}\right)\right] .
$$

Of course, the same result is obtained by taking the limit $\nu_{0} \tau \rightarrow \infty$ in Eq. (32). Equation (35) also allows us to get the moments $\left\langle\xi_{x}^{2 k_{1}} \xi_{\perp}^{2 k_{2}}\right\rangle$. A simple calculation yields

$$
\left\langle\xi_{x}^{2 k_{1}} \xi_{\perp}^{2 k_{2}}\right\rangle=\frac{\Gamma\left(k_{1}+\frac{1}{2}\right) \Gamma\left(k_{2}+\frac{d-1}{2}\right)}{\Gamma\left(\frac{1}{2}\right) \Gamma\left(\frac{d-1}{2}\right)}\left[1+k_{1}\left(2 a^{*}-\lambda\right)-k_{2} \lambda\right]^{-1}
$$

if $1+k_{1}\left(2 a^{*}-\lambda\right)-k_{2} \lambda>0$, being $\infty$ otherwise. The sign of $2 a^{*}-\lambda=(d-1) \lambda /(1-d \lambda)$ is the same as that of $a^{*}$. Thus the moment $\left\langle\xi_{x}^{2 k_{1}} \xi_{\perp}^{2 k_{2}}\right\rangle$ diverges when $k_{1}$ is sufficiently low and $k_{2}$ is sufficiently high in the case $a^{*}>0$, while it diverges when $k_{1}$ is sufficiently high and $k_{2}$ is sufficiently low in the opposite case $a^{*}<0$. More specifically, $\left\langle\xi_{\perp}^{2 k}\right\rangle \rightarrow \infty$ for $a^{*}>0$ if $k \geq 1 / \lambda\left(a^{*}\right)>d$; analogously, $\left\langle\xi_{x}^{2 k}\right\rangle \rightarrow \infty$ for $a^{*}<0$ if $k \geq 1 /\left|2 a^{*}-\lambda\right|>d /(d-1)$. In the particular case of the fourth-degree moments, $\left\langle\xi_{x}^{2} \xi_{\perp}^{2}\right\rangle$ and $\left\langle\xi_{\perp}^{4}\right\rangle$ remain finite but $\left\langle\xi_{x}^{4}\right\rangle$ diverges if $\lambda$ is equal to or smaller than a critical value $\lambda_{c}=-1 /(d-2)$, i.e., if $a^{*} \leq a_{c}^{*}=-d / 4(d-2)$. This behavior is reminiscent of the one observed in Sec. II from the Boltzmann equation, although there are two main differences: (i) in the case of the Boltzmann equation the three fourth-degree moments (i.e., not only $\left\langle\xi_{x}^{4}\right\rangle$ ) diverge and (ii) that happens for a larger departure from equilibrium $\left(a_{c}^{*} \simeq-1.599\right.$ versus $a_{c}^{*}=-0.75$ for $\left.d=3\right)$. The dependence of the three moments on $a^{*}$, as predicted by the BGK model, is shown in Fig. 1. It can be observed a good agreement with the results obtained from the Boltzmann equation in the region $a^{*} \gtrsim-0.3$, especially in the case of $\left\langle\xi_{\perp}^{4}\right\rangle$. For longitudinal rates $a^{*} \lesssim-0.3$, however, the deviations become important.

It is remarkable that in this viscous longitudinal problem the BGK model is able to capture, at least at a qualitative level, the existence of diverging moments for values of $\left|a^{*}\right|$ sufficiently large. In the case of the uniform shear flow, however, all the moments predicted by the BGK equation are finite [25,26], in contrast to the scenario arising from the Boltzmann equation 20 23]. The origin of diverging moments can be traced back to the existence of a high-velocity tail in the distribution function. To clarify this point, let us consider the two marginal distribution functions

$$
\begin{aligned}
\varphi_{\|}\left(\xi_{x} ; a^{*}\right) & \equiv \int \mathrm{d} \boldsymbol{\xi}_{\perp} \Phi\left(\boldsymbol{\xi} ; a^{*}\right) \\
& =\pi^{-1 / 2} \int_{0}^{\infty} \mathrm{d} s \exp \left[-\left(1-a^{*}+\frac{1}{2} \lambda\right) s-e^{(2 a-\lambda) s} \xi_{x}^{2}\right], \\
\varphi_{\perp}\left(\boldsymbol{\xi}_{\perp} ; a^{*}\right) & \equiv \int_{-\infty}^{\infty} \mathrm{d} \xi_{x} \Phi\left(\boldsymbol{\xi} ; a^{*}\right) \\
& =\pi^{-(d-1) / 2} \int_{0}^{\infty} \mathrm{d} s \exp \left[-\left(1+\frac{d-1}{2} \lambda\right) s-e^{-\lambda s} \xi_{\perp}^{2}\right],
\end{aligned}
$$

where $\boldsymbol{\xi}_{\perp} \equiv \boldsymbol{\xi}-\xi_{x} \widehat{\mathbf{x}}$ is the transverse velocity vector. A simple change of variable in Eq. (37) gives 


$$
\varphi_{\|}\left(\xi_{x} ; a^{*}\right)=\pi^{-1 / 2} \frac{F_{\|}\left(a^{*}, \xi_{x}^{2}\right)}{\left|2 a^{*}-\lambda\right|} \xi_{x}^{-2 \beta_{\|}}, \quad \beta_{\|}\left(a^{*}\right) \equiv \frac{1}{2}-\frac{1}{2 a^{*}-\lambda},
$$

where

$$
F_{\|}\left(a^{*}, \xi_{x}^{2}\right)= \begin{cases}\Gamma\left(\beta_{\|}, \xi_{x}^{2}\right), & a^{*}>0 \\ \Gamma\left(\beta_{\|}\right)-\Gamma\left(\beta_{\|}, \xi_{x}^{2}\right), & a^{*}<0\end{cases}
$$

Here,

$$
\Gamma(\beta, x)=\int_{x}^{\infty} \mathrm{d} y y^{\beta-1} e^{-y}
$$

is the incomplete gamma function [27]. Analogously,

$$
\varphi_{\perp}\left(\boldsymbol{\xi}_{\perp} ; a^{*}\right)=\pi^{-(d-1) / 2} \frac{F_{\perp}\left(a^{*}, \xi_{\perp}^{2}\right)}{|\lambda|} \xi_{\perp}^{-2 \beta_{\perp}}, \quad \beta_{\perp}\left(a^{*}\right) \equiv \frac{d-1}{2}+\frac{1}{\lambda},
$$

where

$$
F_{\perp}\left(a^{*}, \xi_{\perp}^{2}\right)= \begin{cases}\Gamma\left(\beta_{\perp}\right)-\Gamma\left(\beta_{\perp}, \xi_{\perp}^{2}\right), & a^{*}>0 \\ \Gamma\left(\beta_{\perp}, \xi_{\perp}^{2}\right), & a^{*}<0\end{cases}
$$

From Eqs. (39) and (40) it follows that $\varphi_{\|} \sim \xi_{x}^{-2 \beta_{\|}}$in the limit $\xi_{x}^{2} \rightarrow \infty$ if $a^{*}<0$, and so $\left\langle\xi_{x}^{2 k}\right\rangle \rightarrow \infty$ if $k \geq \beta_{\|}-\frac{1}{2}=$ $\left|2 a^{*}-\lambda\right|^{-1}$. Similarly, $\varphi_{\perp} \sim \xi_{\perp}^{-2 \beta_{\perp}}$ in the limit $\xi_{\perp}^{2} \rightarrow \infty$ if $a^{*}>0$ and then $\left\langle\xi_{\perp}^{2 k}\right\rangle \rightarrow \infty$ if $k \geq \beta_{\perp}-\frac{d-1}{2}=\lambda^{-1}$. It is interesting to note that the exponents $\beta_{\|}$and $\beta_{\perp}$ remain finite in the limit of infinite $\left|a^{*}\right|: \lim _{a^{*} \rightarrow \infty} \beta_{\perp}\left(a^{*}\right)=$ $(d-1) \lim _{a^{*} \rightarrow-\infty} \beta_{\|}\left(a^{*}\right)=(3 d-1) / 2$ and $\lim _{a^{*} \rightarrow-\infty} \beta_{\perp}\left(a^{*}\right)=(d-1) \lim _{a^{*} \rightarrow \infty} \beta_{\|}\left(a^{*}\right)=(d-1) / 2$.

Apart from an algebraic high-velocity tail, the distribution function may exhibit a singular behavior in the opposite limit of vanishing velocities. Equation (35) shows that $\lim _{\boldsymbol{\xi} \rightarrow \mathbf{0}} \Phi\left(\boldsymbol{\xi} ; a^{*}\right)=\infty$ if $1-a^{*}+\frac{d}{2} \lambda\left(a^{*}\right) \leq 0$, which corresponds to $a^{*} \leq-[\sqrt{d(3 d-2)}-d+2] / 2(d-1)$ and $a^{*} \geq[\sqrt{d(3 d-2)}+d-2] / 2(d-1)$. A similar phenomenon of overpopulation of "rest" particles occurs in the uniform shear flow state [25]. Again, it is useful to consider the marginal distributions in the analysis of this effect. In the case of $\varphi_{\|}$the divergence happens when both $\beta_{\|}$and $a^{*}$ are positive, i.e., for $a^{*}>3 d /(3 d-1)$, while in the case of $\varphi_{\perp}$ the singular behavior takes place when $\beta_{\perp}>0$ and $a^{*}<0$, i.e., for $a^{*}<-d(d+1) /(d-1)(3 d-1)$. By using the properties [25,27]

$$
\lim _{x \rightarrow 0^{+}} \Gamma(\beta, x)= \begin{cases}\Gamma(\beta)-\beta^{-1} x^{\beta}, & \beta>0, \\ -\beta^{-1} x^{\beta}, & \beta<0 \\ -\ln x, & \beta=0\end{cases}
$$

one gets from Eqs. (39) and (42) the following asymptotic behaviors:

$$
\begin{aligned}
& \lim _{\xi_{x}^{2} \rightarrow 0} \varphi_{\|}\left(\xi_{x} ; a^{*}\right)= \begin{cases}\pi^{-1 / 2}\left(1-a^{*}+\frac{1}{2} \lambda\right)^{-1}, & a^{*}<\frac{3 d}{3 d-1}, \\
\pi^{-1 / 2} \frac{\Gamma\left(\beta_{\|}\right)}{2 a^{*}-\lambda} \xi_{x}^{-2 \beta_{\|},}, & a^{*}>\frac{3 d}{3 d-1}, \\
\pi^{-1 / 2} \ln \left|\xi_{x}\right|^{-1}, & a^{*}=\frac{3 d}{3 d-1},\end{cases}
\end{aligned}
$$

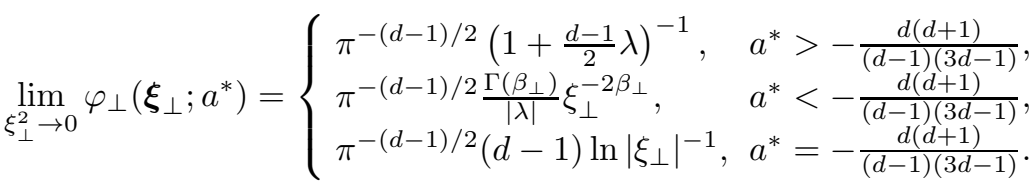

Figure 2 shows the ratios with respect to local equilibrium $R_{\|}\left(\xi_{x} ; a^{*}\right)=\varphi_{\|}\left(\xi_{x} ; a^{*}\right) / \varphi_{\|}\left(\xi_{x} ; 0\right)$ and $R_{\perp}\left(\boldsymbol{\xi}_{\perp} ; a^{*}\right)=$ $\varphi_{\perp}\left(\boldsymbol{\xi}_{\perp} ; a^{*}\right) / \varphi_{\perp}\left(\boldsymbol{\xi}_{\perp} ; 0\right)$ for $a^{*}=-1$ and $a^{*}=1.5$ in the three-dimensional case. It can be observed that at $a^{*}=-1$ $\left(a^{*}=1.5\right)$ the function $\varphi_{\|}\left(\varphi_{\perp}\right)$ develops a high-velocity tail, while the function $\varphi_{\perp}\left(\varphi_{\|}\right)$diverges as the velocity vanishes. 


\section{B. Repulsive potentials. Hard spheres}

Now we consider more general repulsive potentials characterized by $\gamma>0$. The BGK model for our problem is given by Eq. (31). Its general solution is

$$
\begin{aligned}
\tilde{f}(\mathbf{V}, \tau)= & e^{-s(\tau)} e^{a \tau \partial_{V_{x}} V_{x}} \tilde{f}(\mathbf{V}, 0) \\
& +\nu_{0} \int_{0}^{\tau} \mathrm{d} \tau^{\prime}\left[\frac{T\left(\tau^{\prime}\right)}{T_{0}}\right]^{\gamma} e^{-\left[s(\tau)-s\left(\tau^{\prime}\right)\right]} e^{a\left(\tau-\tau^{\prime}\right) \partial_{V_{x}} V_{x}} \widetilde{f}_{L}\left(\mathbf{V}, \tau^{\prime}\right),
\end{aligned}
$$

where

$$
s(\tau)=\nu_{0} \int_{0}^{\tau} \mathrm{d} \tau^{\prime}\left[\frac{T\left(\tau^{\prime}\right)}{T_{0}}\right]^{\gamma}
$$

is the number of collisions per particle. In the limit $\gamma \rightarrow 0, s(\tau)=\nu_{0} \tau$ and Eq. (47) reduces to Eq. (32). On the other hand, for $\gamma>0 \mathrm{Eq}$. (47) is not closed since it requires the knowledge of the time dependence of the temperature. By multiplying both sides of Eq. (47) by $V^{2}$ and integrating over velocity one can get a closed integral equation for $T(\tau)$. Such an equation is however quite involved and then it is more transparent to work directly with the evolution equation itself, Eq. (31). From this equation it is straightforward to find that the evolution of $\widetilde{p}$ and $\widetilde{P}_{x x}$ is still given by Eqs. (9) and (10), except that now $\nu_{0}$ is replaced by a time-dependent collision frequency $\nu_{0}\left[\widetilde{p}(\tau) / p_{0}\right]^{\gamma}$. Therefore, the pressure $\widetilde{p}=n_{0} k_{B} T$ obeys a nonlinear second-order differential equation

$$
\frac{\partial^{2}}{\partial \tau^{2}} \widetilde{p}+\left[2 a+\nu_{0}\left(\frac{\tilde{p}}{p_{0}}\right)^{\gamma}\right] \frac{\partial}{\partial \tau} \widetilde{p}+\frac{2}{d} a \nu_{0}\left(\frac{\tilde{p}}{p_{0}}\right)^{\gamma} \widetilde{p}
$$

subject to the initial conditions $\widetilde{p}(0)=p_{0}, \partial \widetilde{p} /\left.\partial \tau\right|_{\tau=0}=-(2 a / d) P_{x x}(0)$. For asymptotically long times $(\tau \rightarrow \infty)$, the solution of Eq. (49) behaves as $\widetilde{p}(\tau) \sim \exp (-2 a \tau / d)$ if $a<0$ and as $\widetilde{p}(\tau) \sim\left(1+\gamma \nu_{0} \tau / d\right)^{-1 / \gamma}$ if $a>0$. In both cases the accumulated number of collisions $s(\tau)$, Eq. (48), goes to infinity as $\tau \rightarrow \infty$. In particular, if $a<0$, the typical number of collisions per particle during the finite interval $0 \leq t \leq|a|^{-1}$ becomes infinite, a property already seen in Sec. III for Maxwell molecules.

In order to get the nonlinear viscosity as a function of the longitudinal deformation rate, we must work with the reduced rate $a^{*}=A / \nu=a / \nu_{0}\left(\widetilde{p} / p_{0}\right)^{\gamma}$ rather than with the time variables $\tau$ or $t$. Note that $\lim _{\tau \rightarrow \infty} a^{*}(\tau)=\infty$ if $a>0$, while $\lim _{\tau \rightarrow \infty} a^{*}(\tau)=0$ if $a<0$. With this change of variable one has

$$
\begin{gathered}
\frac{\partial}{\partial a^{*}} \widetilde{p}=-\frac{\widetilde{p}}{\gamma a^{*}} \\
\frac{\partial}{\partial a^{*}} \widetilde{P}_{x x}=-\frac{d \widetilde{p}}{\gamma a^{*}}\left[1+\frac{1}{2 a^{*}}\left(1-\frac{\widetilde{p}}{\widetilde{P}_{x x}}\right)\right] .
\end{gathered}
$$

This gives rise to the following ordinary differential equation for the reduced nonlinear viscosity defined in Eq. (14):

$$
2 \gamma a^{* 2}\left(1-2 \frac{d-1}{d} a^{*} \eta^{*}\right) \frac{\partial \eta^{*}}{\partial a^{*}}+4 \frac{d-1}{d}(1-\gamma) a^{* 2} \eta^{* 2}+\left[d+2(d-2+\gamma) a^{*}\right] \eta^{*}-d=0 .
$$

For small $a^{*}$ the solution is $\eta^{*} \approx 1-[2(d-2+\gamma) / d] a^{*}$, while the asymptotic behavior of $\eta^{*}$ for large $\left|a^{*}\right|$ is $\eta^{*} \approx[d / 2(d-1)] a^{*-1}\left(1-a^{*-1} / 2\right)$ for $a^{*}>0$ and $\eta^{*} \approx(d / 2)\left|a^{*}\right|^{-1}\left[1-\left|a^{*}\right|^{-1} / 2(1+\gamma)\right]$ for $a^{*}<0$. The leading terms are independent of $\gamma$ and correspond to $\lim _{a^{*} \rightarrow+\infty} P_{x x} / p=0$ and $\lim _{a^{*} \rightarrow-\infty} P_{x x} / p=d$, respectively. Interestingly enough, Eq. (52), particularized to $d=3$, is equivalent to the one derived by Karlin et al. [9] from their invariance principle and Grad's method. In other words, the invariance principle under the microscopic and macroscopic dynamics is an approximation that, at least in this problem, yields the same nonlinear viscosity as the one predicted by the BGK model. The latter approach, nevertheless, has the advantages of being conceptually simpler and providing the full velocity distribution function.

It must be noted that Eq. (52) possesses as many solutions as particular initial conditions. Each particular solution is specified by assigning a given value of the viscosity $\eta^{*}$ at the initial (reduced) longitudinal rate $a_{0}^{*}=a / \nu_{0}$. This situation is analogous to the one discussed in Ref. 25] for the uniform shear flow case. Since the irreversible time evolution of the system leads to a monotonic increase of $a^{*}$, Eq. (52) must be solved for $a^{*} \geq a_{0}^{*}$. For positive rates, 
this implies the range $0<a_{0}^{*} \leq a^{*}$, but for negative rates the range is $a_{0}^{*} \leq a^{*}<0$. All the particular solutions, however, tend towards a special solution (the hydrodynamic one) for sufficiently long times, i.e., for $a^{*} \gg a_{0}^{*}$ if $a_{0}^{*}>0$ and for $\left|a^{*}\right| \ll\left|a_{0}^{*}\right|$ if $a_{0}^{*}<0$. This special solution representing the hydrodynamic or normal regime can be identified in principle by the Chapman-Enskog series (16). Insertion into the differential equation (52) yields the following recurrence formula,

$$
c_{n}=-2 \frac{d-2+n \gamma}{d} c_{n-1}-4 \frac{d-1}{d^{2}} \sum_{m=0}^{n-2} c_{m} c_{n-2-m}[1-(n-1-m) \gamma] .
$$

This equation shows that the coefficient $c_{n}$ is a polynomial in $\gamma$ of degree $n$. The first four coefficients in the case of a three-dimensional system of hard spheres $\left(d=3, \gamma=\frac{1}{2}\right)$ are $c_{1}=-1, c_{2}=\frac{8}{9}, c_{3}=-\frac{28}{27}$, and $c_{4}=\frac{56}{27}$. Further computation of the coefficients shows that, except in the case of Maxwell molecules $(\gamma=0)$, the expansion (16) is only asymptotic. For large $n$, the magnitude of the coefficients $c_{n}$ grow so rapidly that the second term on the right-hand-side of Eq. (53) can be neglected, so $c_{n} / c_{n-1} \approx-2 n \gamma / d$. The ratio $-c_{n} / c_{n-1}, n=1-20$, is plotted in Fig. 3 for three-dimensional hard spheres. The linear growth of the ratio is already apparent for $n \geq 4$. The divergence of the Chapman-Enskog expansion for $\gamma>0$ also takes place in the uniform shear flow problem 25,28$]$.

Since the series (16) is only useful if truncated and applied to small $a^{*}$, a different strategy is needed to get the hydrodynamic $\eta^{*}$ for finite $a^{*}$. One possibility is to expand $\eta^{*}$ around the point at infinity (namely in powers of $a^{*-1}$ ). Such an expansion proved to be convergent in the case of uniform shear flow for shear rates larger than a certain value [25,28. From a practical point of view, however, this method is not very convenient because many terms would need to be retained in order to get reliable results in the range of interest (say $\left|a^{*}\right| \sim 1$ ), even if the expansion converges. A second possibility is to solve numerically the differential equation (52) with the boundary conditions $\lim _{a_{0}^{*} \rightarrow 0^{+}} \eta^{*}\left(a_{0}^{*}\right)=1$ (for $a^{*}>0$ ) and $\lim _{a_{0}^{*} \rightarrow-\infty} \eta^{*}\left(a_{0}^{*}\right)=0$ (for $a^{*}<0$ ). On the other hand, it seems more convenient to follow the approach proposed in Ref. [9], which consists of representing the nonlinear viscosity as an expansion in powers of the interaction parameter $\gamma$ :

$$
\eta^{*}\left(a^{*}\right)=\lim _{N \rightarrow \infty} \eta^{(N)}\left(a^{*}\right), \quad \eta^{(N)}\left(a^{*}\right)=\sum_{n=0}^{N} \eta_{n}\left(a^{*}\right) \gamma^{n},
$$

where $\eta_{0}\left(a^{*}\right)$ is the nonlinear viscosity for Maxwell molecules, Eq. (15). Since the Chapman-Enskog coefficients $c_{n}$ are polynomials in $\gamma$, the series (54) can be interpreted as a rearrangement of the series (16). In other words, if we write

$$
c_{n}=\sum_{m=0}^{n} c_{n m} \gamma^{m}
$$

then

$$
\eta_{n}\left(a^{*}\right)=\sum_{m=n}^{\infty} c_{m n} a^{* m}
$$

As a consequence, the truncated series $\eta^{(N)}\left(a^{*}\right)$ is exact through order $a^{* N}$, i.e., $\eta^{*}\left(a^{*}\right)-\eta^{(N)}\left(a^{*}\right)=\mathcal{O}\left(a^{* N+1}\right)$. Insertion of Eq. (54) into Eq. (52) yields the following recurrence formula

$$
\begin{aligned}
\eta_{n}\left(a^{*}\right)= & -\frac{2 a}{d+2(d-2) a^{*}+8 \frac{d-1}{d} a^{* 2} \eta_{0}\left(a^{*}\right)}\left\{\left[1-2 \frac{d-1}{d} a^{*} \eta_{0}\left(a^{*}\right)\right]\left[\eta_{n-1}\left(a^{*}\right)+a^{*} \eta_{n-1}^{\prime}\left(a^{*}\right)\right]\right. \\
& \left.-2 \frac{d-1}{d} a^{*} \sum_{m=1}^{n-1} \eta_{m}\left(a^{*}\right)\left[\eta_{n-1-m}\left(a^{*}\right)+a^{*} \eta_{n-1-m}^{\prime}\left(a^{*}\right)-\eta_{n-m}\left(a^{*}\right)\right]\right\},
\end{aligned}
$$

where the prime denotes a derivative with respect to $a^{*}$. The coefficient $\eta_{1}$ for $d=3$ was the only one considered by Karlin et al. [9,11,29]. The coefficients $\eta_{n}$ for $n=0,1,3,5,6$ and $d=3$ are plotted in Fig. 1 . Up to $n=3$ the coefficients remain small, but the magnitude of $\eta_{5}$ and, especially, that of $\eta_{6}$ reach rather high values, thus suggesting the asymptotic character of the expansion (54), at least for negative $a^{*}$. This is not surprising if one takes into account that, while all the truncated series $\eta^{(N)}\left(a^{*}\right)$ are regular at $a^{*}=0$, the full viscosity $\eta^{*}\left(a^{*}\right)$ is singular at $a^{*}=0$. Notwithstanding this, since the maximum value of $\gamma$ is $\gamma=\frac{1}{2}$, it turns out that the functions $\eta^{(N)}\left(a^{*}\right)$ with $N=3$ or $N=4$ can be considered as rather good approximations of $\eta^{*}\left(a^{*}\right)$. This is quite apparent in Fig. 5 , 
where $\eta^{(3)}\left(a^{*}\right)$ and $\eta^{(4)}\left(a^{*}\right)$ practically overlap in the case $\gamma=\frac{1}{3}$ (corresponding to a repulsive potential with $\mu=12$ ) and are hardly distinguishable in the case of $\gamma=\frac{1}{2}$ (hard spheres). Figure 5 also shows that the nonlinear viscosity is almost insensitive to the interaction model in the case of an expansion $\left(a^{*}>0\right)$. On the other hand, when the physical situation corresponds to a condensation of the gas $\left(a^{*}<0\right)$, the hardness of the repulsion plays a relevant role, especially around the maximum $\left(a^{*} \approx-0.4\right)[30]$.

Before closing this Section, it is worthwhile noting that Uribe and García-Colín [12] used Grad's (nonlinear) moment method to get an expression for $\eta^{*}\left(a^{*}\right)$ that is dramatically at odds with the results obtained in this paper for $a^{*}<0$. According to their results, $\eta^{*}\left(a^{*}\right)$ monotonically increases as $a^{*}$ becomes more and more negative and finally reaches a plateau $\eta^{*} \rightarrow 49$ in the limit $a^{*} \rightarrow-\infty$. However, these results are strongly inconsistent with the physical condition $p=\left[P_{x x}+(d-1) P_{y y}\right] / d \geq P_{x x} / d$, which implies [cf. Eq. (14)] that $\eta^{*}\left(a^{*}\right) \leq(d / 2)\left|a^{*}\right|^{-1}$ if $a^{*}<0$. Since $\eta^{*}$ has an upper bound that goes to zero in the limit $a^{*} \rightarrow-\infty$, then $\lim _{a^{*} \rightarrow-\infty} \eta^{*}\left(a^{*}\right)=0$ necessarily. On the other hand, from Eqs. (45) or (47) of Ref. [12 it follows that $p<P_{x x} / 3$ (i.e, $P_{y y}<0$ ) if $a^{*}<-\frac{5}{14}$. These inconsistencies of the results derived in Ref. 12] are likely associated with the assumption of a stationary situation in this compressible flow.

\section{CONCLUSIONS}

This paper has dealt with a simple viscous longitudinal flow characterized by an unsteady velocity profile $u_{x}(x, t)=$ $a x /(1+a t)$ and a uniform density $n(t)=n_{0} /(1+a t)$. The situation with $a>0$ corresponds to an expansion of the gas, while the case $a<0$ describes a condensation phenomenon. By using kinetic theory tools (Boltzmann equation and BGK kinetic model), exact results have been derived for the generalized or nonlinear viscosity, the velocity moments, and the velocity distribution function. The following points summarize the main conclusions of the present study.

- By an adequate change of velocity and time variables, the problem is seen to be formally equivalent to that of a uniform gas with a steady density, in which the particles are under the action of a longitudinal driving force $\mathbf{F}=-m a V_{x} \widehat{\mathbf{x}}$. According to this viewpoint, the particles are decelerated or accelerated along the longitudinal direction, depending on the sign of $a$. As a consequence, the temperature monotonically decreases in time if $a>0$, while it increases if $a<0$.

- The relative difference between the normal stress $P_{x x}$ and the hydrostatic pressure $p$ for long collision times is characterized by a (dimensionless) viscosity coefficient $\eta^{*}\left(a^{*}\right)$, which is a nonlinear function of the longitudinal deformation rate relative to an effective collision frequency. The (Chapman-Enskog) expansion of $\eta^{*}$ in powers of $a^{*}$ is, in general, only asymptotic. An exception is provided by Maxwell molecules, in which case the expansion converges for $\left|a^{*}\right|<\frac{1}{2}$.

- A thinning effect is present for $a^{*}>0$, i.e., $\eta^{*}$ monotonically decreases as $a^{*}$ increases. For $a^{*}<0$, however, $\eta^{*}$ starts increasing with $\left|a^{*}\right|$ (thickening effect), reaches a maximum (at $a^{*}=-\frac{1}{3}$ for Maxwell molecules and around $a^{*} \simeq-0.4$ for hard spheres), and then decreases for more negative longitudinal rates.

- In the case of an expansion $\left(a^{*}>0\right)$, the nonlinear viscosity $\eta^{*}$ is practically "universal". On the other hand, its behavior in the case of condensation $\left(a^{*}<0\right)$ is rather sensitive to the interaction potential. In particular, the harder the potential the higher the maximum value of $\eta^{*}$ (for instance, $\eta_{\max }^{*}=1.125$ for Maxwell molecules and $\eta_{\max }^{*} \simeq 1.46$ for hard spheres).

- The results for $\eta^{*}$ derived from the Boltzmann equation for Maxwell molecules and from the BGK model for more general potentials coincide with those derived by Karlin et al. [9] from Grad's method and the application of their invariance principle under microscopic and macroscopic dynamics. It would be interesting to explore whether such an equivalence extends to the similar but more complicated problem of uniform shear flow as well.

- The shape of the velocity distribution function (for Maxwell molecules) has also been analyzed by scaling the velocities with the (unsteady) thermal velocity. This gives rise to a new term in the kinetic equation that represents a non-conservative thermostat force that cancels the cooling $\left(a^{*}>0\right)$ or heating $\left(a^{*}<0\right)$ produced by the driving force. This exact equivalence between the free system and the thermostatted one is analogous to that taking place in the uniform shear flow and is restricted to Maxwell molecules (collision frequency independent of the velocity). For other interactions the equivalence is only approximate, but yet the thermostatted problem is worth studying by itself. It would be quite interesting to carry out nonequilibrium molecular dynamics simulations of hard spheres subject to the simultaneous action of the driving and thermostat forces, in order to measure the nonlinear viscosity and related phenomena in dense gases. This would complement the extensive simulation studies of the uniform shear flow. 
- The exact fourth-degree (scaled) velocity moments derived from the Boltzmann equation diverge for sufficiently negative values of the longitudinal rate $\left(a^{*} \lesssim-1.6\right)$. This indicates the existence of an algebraic high-velocity tail, especially for the longitudinal component of the velocity, for negative $a^{*}$.

- The above singular behavior of the moments is described, at least qualitatively, by the exact solution of the BGK model. This is a very remarkable feature, since a similar behavior showing up in the uniform shear flow was not captured by the kinetic model. Also, note that Grad's method is unable to predict an algebraic high-velocity tail, as it approximates the distribution function by a Gaussian times a polynomial. The BGK solution obtained in this paper predicts an algebraic tail in the marginal distribution of longitudinal velocities if $a^{*}<0$ and a weaker tail in the marginal distribution of transverse velocities if $a^{*}>0$. The latter tail implies that, while all the moments of degrees equal to or smaller than six (for a three-dimensional system) are finite when $a^{*}>0$, the moments of eighth degree diverge if $a^{*} \geq 1.125$. The investigation of whether or not this prediction is confirmed by the Boltzmann equation will be the subject of a separate paper.

- The explicit expression of the BGK velocity distribution function allows one to unveil a different type of singular behavior that does not have, however, a direct influence on the velocity moments since it is associated with the limit of small velocities. More specifically, the distribution of vanishing velocities diverges if the longitudinal rate is sufficiently positive $\left(a^{*} \gtrsim 1.40\right)$ or sufficiently negative $\left(a^{*} \lesssim-0.90\right)$. This effect is also present in the marginal distribution of longitudinal velocities (for $a^{*} \geq 1.125$ ) and in that of transverse velocities (for $a^{*} \leq-0.75$ ).

\section{ACKNOWLEDGMENTS}

The author acknowledges partial support from the DGES (Spain) through grant No. PB97-1501 and from the Junta de Extremadura (Fondo Social Europeo) through grant No. IPR99C031.

* $\quad$ Electronic mail: andres@unex.es

[1] S. R. de Groot and P. Mazur, Nonequilibrium Thermodynamics (Dover, New York, 1984).

[2] See, for instance, B. C. Eu, Nonequilibrium Statistical Mechanics (Kluwer Academic Publishers, Dordrecht, 1998) and references therein.

[3] J. W. Dufty, J. J. Brey, and A. Santos, in Molecular-Dynamics Simulation of Statistical-Mechanical Systems, edited by G. Ciccotti and W. G. Hoover (North-Holland, Amsterdam, 1986), pp. 294-303.

[4] D. J. Evans and G. P. Morriss, Statistical Mechanics of Nonequilibrium Liquids (Academic Press, London, London, 1990).

[5] J. F. Lutsko, Phys. Rev. Lett. 78, 243 (1997).

[6] J. F. Lutsko, Phys. Rev. E 58, 434 (1997).

[7] A. Santos, J. M. Montanero, J. W. Dufty, and J. J. Brey, Phys. Rev. E 57, 1644 (1998).

[8] A. N. Gorban and I. V. Karlin, Phys. Rev. Lett. 77, 282 (1996).

[9] I. V. Karlin, G. Dukek, and T. F. Nonnenmacher, Phys. Rev. E 55, 1573 (1997).

[10] F. J. Uribe and E. Piña, Phys. Rev. E 57, 3672 (1998).

[11] I. V. Karlin, G. Dukek, and T. F. Nonnenmacher, Phys. Rev. E 57, 3674 (1998).

[12] F. J. Uribe and L. S. García-Colín, Phys. Rev. E 60, 4052 (1999).

[13] C. Truesdell and R. G. Muncaster, Fundamentals of Maxwell's Kinetic Theory of a Simple Monatomic Gas (Academic Press, New York, 1980).

[14] V. S. Galkin, Fluid Dynamics 30, 467 (1995).

[15] S. Chapman and T. G. Cowling, The Mathematical Theory of Nonuniform Gases (Cambridge University Press, Cambridge, 1970).

[16] J. W. Dufty, in Lectures on Thermodynamics and Statistical Mechanics, edited by M. L. de Haro and C. Varea (World Scientific, Singapore, 1990), pp. 166-181.

[17] M. H. Ernst, Phys. Rep. 78, 1 (1981).

[18] J. R. Dorfman and H. van Beijeren, in Statistical Mechanics, Part B, edited by B. J. Berne (Plenum, New York, 1977), pp. $65-179$.

[19] Z. Alterman, K. Frankowski, and C. L. Pekeris, Astrophys. J. Suppl. Ser. 7, 291 (1962).

[20] A. Santos, V. Garzó, J. J. Brey, and J. W. Dufty, Phys. Rev. Lett. 71, 3971 (1993).

[21] A. Santos and V. Garzó, Physica A 213, 409 (1995).

[22] J. M. Montanero, A. Santos, and V. Garzó, Phys. Rev. E 53, 1269 (1996). 
[23] J. M. Montanero, A. Santos, and V. Garzó, J. Stat. Phys. 88, 1165 (1997).

[24] P. L. Bhatnagar, E. P. Gross, and M. Krook, Phys. Rev. 94, 511 (1954).

[25] A. Santos and J. J. Brey, Physica A 174, 355 (1991).

[26] V. Garzó and A. Santos, Physica A 213, 426 (1995).

[27] Handbook of Mathematical Functions, edited by M. Abramowitz and I. A. Stegun (Dover, New York, 1972).

[28] A. Santos, J. J. Brey, and J. W. Dufty, Phys. Rev. Lett. 56, 1571 (1986).

[29] Note a misprint in Eq. (16) of Ref. 9]: The fraction $(3 / 2)$ in the numerator should be replaced by $\left(1-g R_{0}\right)$.

[30] It is worthwhile mentioning that the approximation $\eta^{(1)}$ underestimates the maximum by about $9 \%$ in the case of hard spheres. 


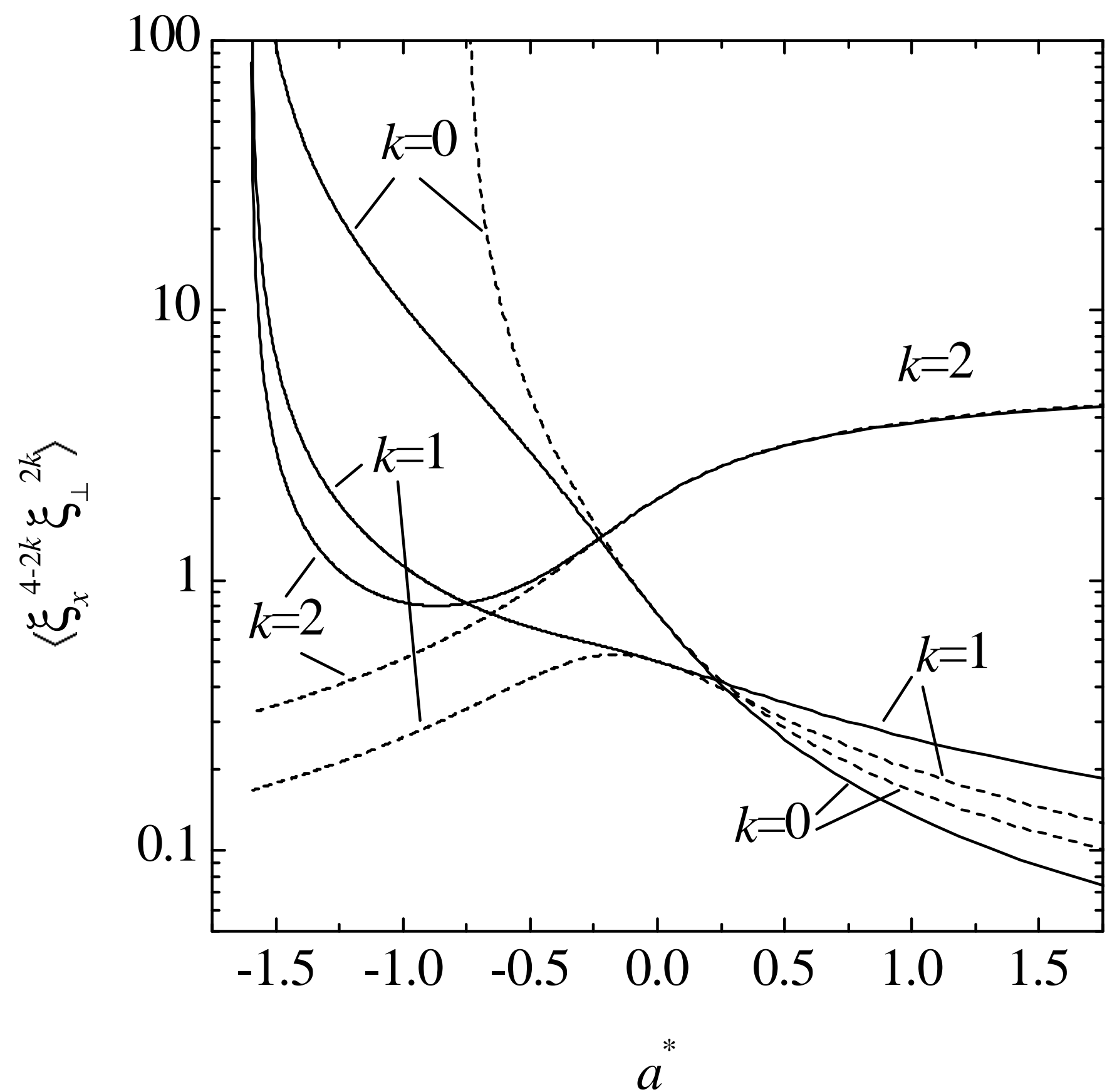

FIG. 1. Plot of the reduced fourth-degree moments $\left\langle\xi_{x}^{4}\right\rangle(k=0),\left\langle\xi_{x}^{2} \xi_{\perp}^{2}\right\rangle(k=1)$, and $\left\langle\xi_{\perp}^{4}\right\rangle(k=2)$ as functions of the reduced longitudinal rate $a^{*}$ in a three-dimensional system of Maxwell molecules. Solid lines refer to the exact results derived from the Boltzmann equation, while dashed lines are predictions of the BGK kinetic model. 


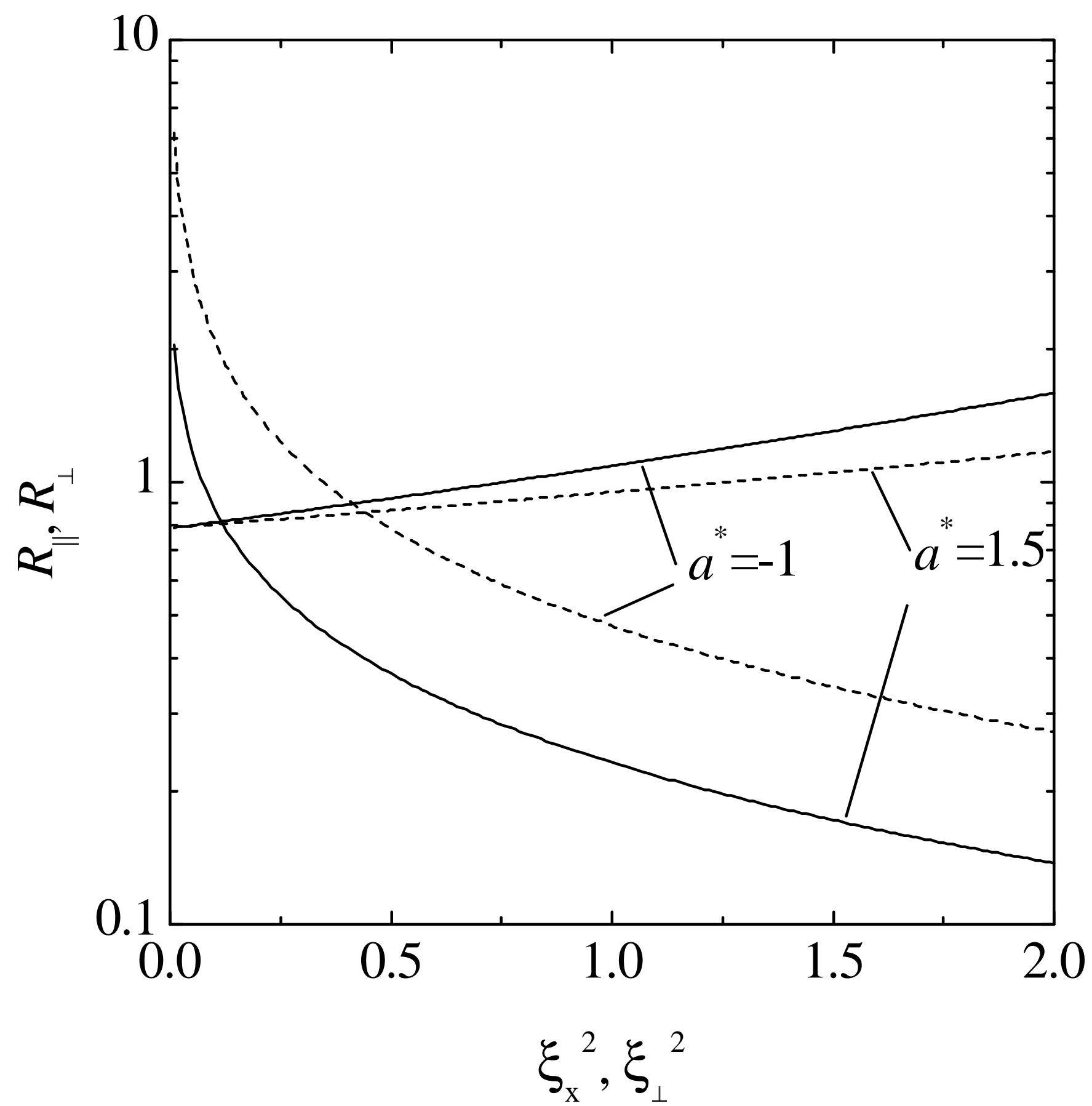

FIG. 2. Marginal velocity distributions normalized with respect to local equilibrium, $R_{\|}\left(\xi_{x}\right)$ (solid lines) and $R_{\perp}\left(\boldsymbol{\xi}_{\perp}\right)$ (dashed lines), for $a^{*}=-1$ and $a^{*}=1.5$, as predicted by the BGK kinetic model for a three-dimensional system of Maxwell molecules. 


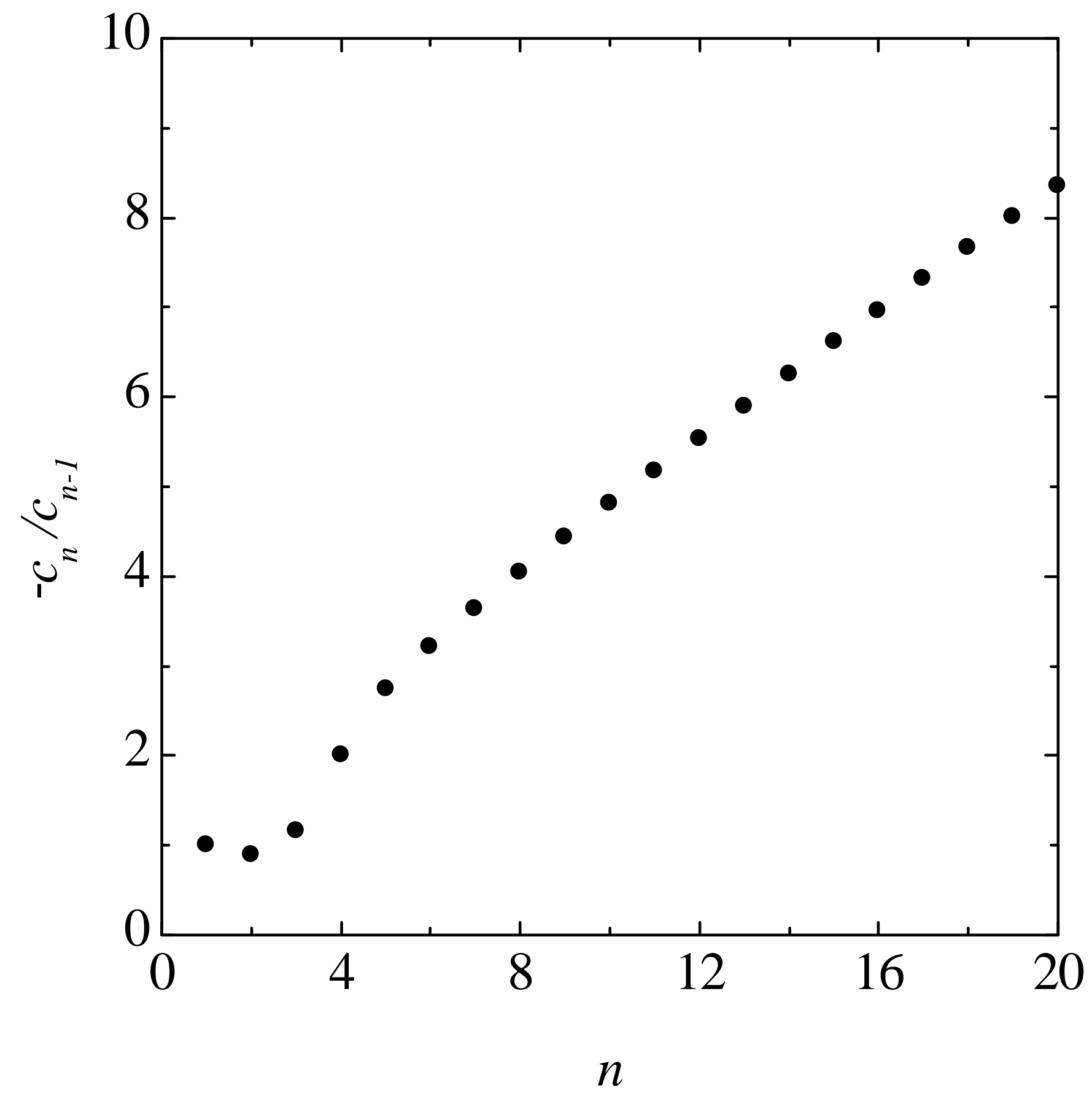

FIG. 3. Ratio $-c_{n} / c_{n-1}$ between successive coefficients in the Chapman-Enskog expansion of the nonlinear viscosity for a three-dimensional system of hard spheres, according to the BGK kinetic model. 


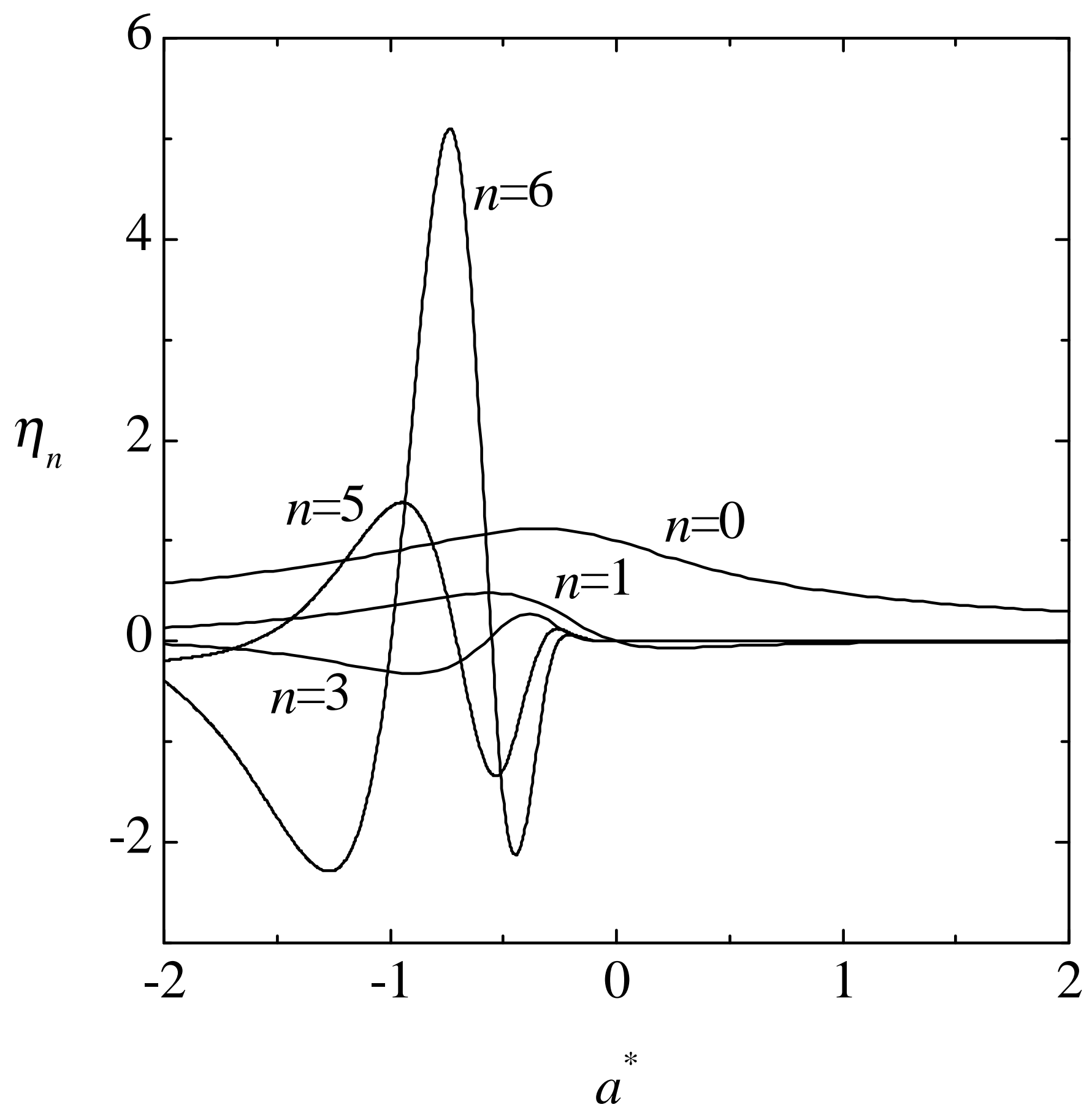

FIG. 4. Longitudinal-rate dependence of the coefficients $\eta_{n}, n=0,1,3,5,6$ of the expansion of the nonlinear viscosity in powers of the interaction parameter $\gamma$, according to the BGK kinetic model for three-dimensional systems. 


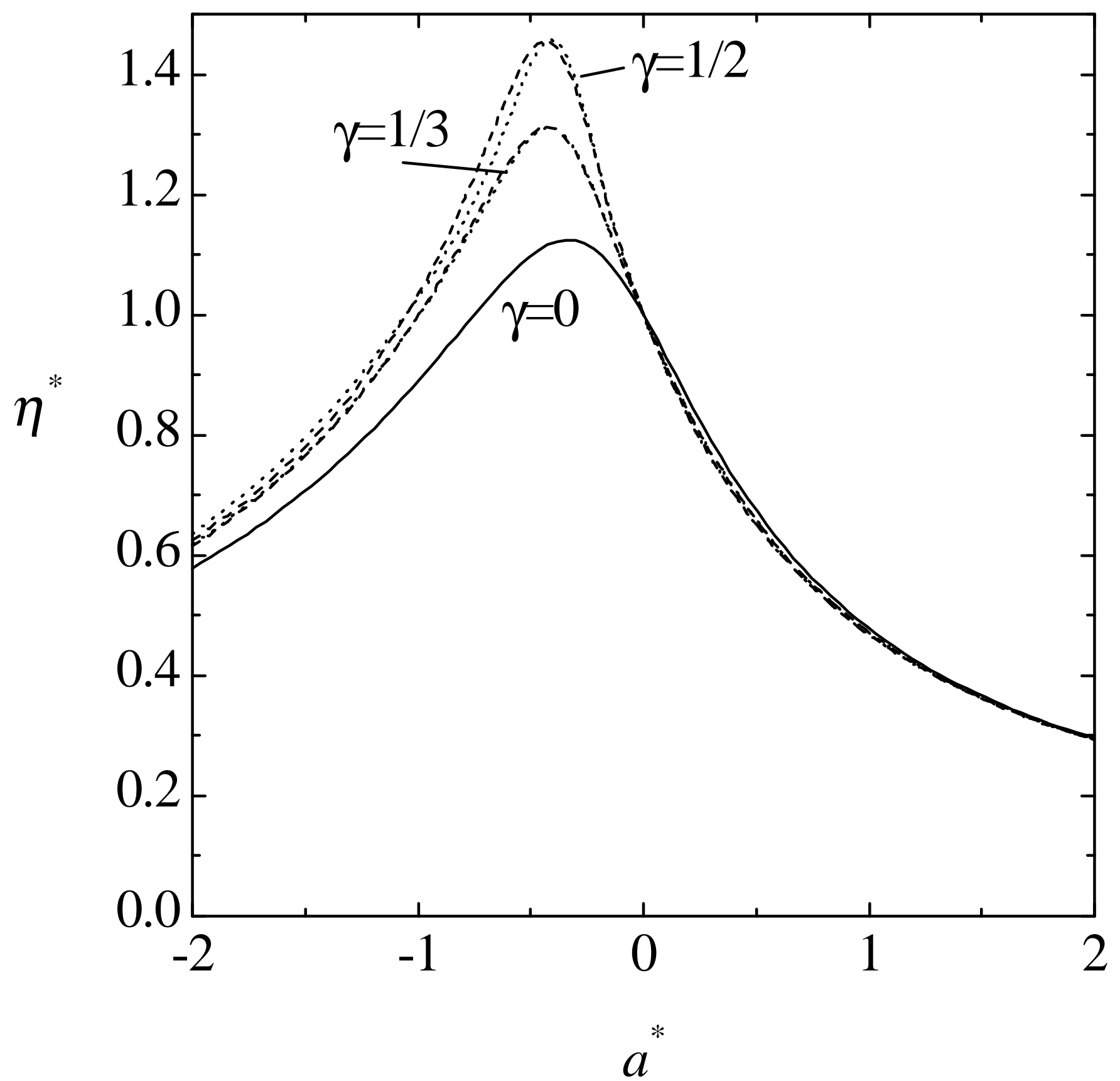

FIG. 5. Nonlinear viscosity for three-dimensional systems of Maxwell molecules $(\gamma=0)$, particles interacting via an $r^{-12}$-potential $\left(\gamma=\frac{1}{3}\right)$, and hard spheres $\left(\gamma=\frac{1}{2}\right)$. The solid line is the exact result derived from the Boltzmann equation and the BGK model, while the dashed and dotted lines are the approximations $\eta^{(3)}$ and $\eta^{(4)}$, respectively, as obtained from the BGK kinetic model. 